\title{
Risco de mortalidade por todas as causas e sua relação com estado de saúde em uma coorte de idosos residentes na comunidade: Estudo FIBRA
}

\author{
Risk of all-cause mortality and its association with health status \\ in a cohort of community-dwelling older people: FIBRA study
}

Priscila Maria Stolses Bergamo Francisco (https://orcid.org/0000-0001-7361-9961) ${ }^{1}$

Daniela de Assumpção (http://orcid.org/0000-0003-1813-996X) ${ }^{1}$

Flávia Silva Arbex Borim (https://orcid.org/0000-0001-7316-1145) ${ }^{1}$

Monica Sanches Yassuda (https://orcid.org/0000-0002-9182-2450) ${ }^{1}$

Anita Liberalesso Neri (https://orcid.org/0000-0002-6833-7668) ${ }^{1}$

${ }^{1}$ Faculdade de Ciências Médicas, Universidade Estadual de Campinas. R. Tessália Vieira de Camargo 126, Cidade Universitária. 13083-887 Campinas SP Brasil. primaria@unicamp.br

\begin{abstract}
This article aims to estimate the risk of death according to sociodemographic characteristics, chronic diseases, frailty, functional capacity, and social participation in older people as well as determine the median time of death in relation to health status and social participation. A retrospective longitudinal study was conducted with older people ( $\geq 65$ years) in 2008-09 and 2016-17 in the city of Campinas and the subdistrict of Ermelino Matarazzo in the city of São Paulo. Face-to-face interviews were conducted at community centers and the participants' homes. The cumulative incidence of death was estimated and associations with the predictor variables were analyzed using Poisson multiple regression. The Kaplan-Meier method and the log-rank test were also used. Among the 741 individuals located at follow-up, 192 had deceased. The incidence of death was higher among those who reported having heart disease and those who were dependent on others regarding the performance of instrumental activities of daily living. The incidence of death was lower among women, individuals in the highest income stratum, and those who performed three or more activities related to social inclusion. No differences in median survival times were found. Predictors of mortality can contribute to broadening knowledge on the singularities of the aging process.
\end{abstract}

Key words Mortality, Aged, Frailty, Activities of daily living, Social participation
Resumo O artigo tem por objetivos estimar o risco de óbito segundo características sociodemográficas, doenças crônicas, fragilidade, capacidade funcional e participação social em idosos e verificar, para as variáveis de estado de saúde e participação social, o tempo mediano de ocorrência do óbito. Estudo longitudinal retrospectivo com idosos ( $\geq 65$ anos), realizado em 2008-09 e 201617 em Campinas-SP e Ermelino Matarazzo-SP. Realizaram-se entrevistas face a face em centros de convivência e nos domicílios. Estimou-se a incidência acumulada de óbito e associações com as variáveis preditoras foram analisadas pela regressão múltipla de Poisson. O método de KaplanMeier e o teste de Log-rank foram utilizados. Dos 741 idosos localizados no seguimento, 192 faleceram. Observou-se maior incidência de óbito nos mais idosos, nos que relataram doença do coração e nos dependentes para realização de atividades instrumentais da vida diária. Verificou-se menor incidência de óbito nas mulheres, no estrato com maior renda e nos que realizavam três ou mais atividades relacionadas à inserção social. Não se observaram diferenças nos tempos medianos de sobrevida. Os preditores de mortalidade podem contribuir para ampliar o conhecimento sobre as singularidades do processo de envelhecimento.

Palavras-chave Mortalidade, Idoso, Fragilidade, Atividades cotidianas, Participação social 


\section{Introdução}

O envelhecimento populacional no Brasil e no mundo é decorrente de alterações demográficas e epidemiológicas, de conquistas científico-tecnológicas que, associadas à melhoria da qualidade de vida, refletiram-se no aumento da longevida$\mathrm{de}^{1,2}$ e da prevalência de doenças crônicas e agravos não transmissíveis ${ }^{2,3}$.

Os idosos brasileiros, assim como outros grupos etários da população, apresentam condições socioeconômicas heterogêneas ${ }^{2,4}$, com marcadas discrepâncias no apoio social, em aspectos psicossociais, no estilo de vida, na capacidade funcional ${ }^{5}$ e no acesso aos serviços de saúde para diagnóstico, tratamento ou controle de doenças crônicas ${ }^{6}$. Neste sentido, amplia-se a necessidade de novas concepções, tecnologias, políticas e modelos de atenção, tendo como foco a promoção e a prevenção da saúde ${ }^{7}$, que possibilitem um envelhecimento saudável.

No que se refere à distribuição dos óbitos na população brasileira, de acordo com o Sistema de Informação sobre Mortalidade (SIM), cerca de $60 \%$ do total ocorre entre idosos com idade $\geq 65$ anos, com destaque para os idosos do sexo feminino $(68,5 \%)$ em relação ao masculino $(52,4 \%)$, particularmente na faixa etária de 65 a 74 anos. Quanto às causas específicas de morte, os registros de óbitos para 2018 apontaram que, nos idosos brasileiros com 65 anos ou mais, $65 \%$ das mortes foram provocadas por doenças do aparelho circulatório, seguidas das neoplasias (tumores) e doenças do aparelho respiratório ${ }^{8}$, como observado em outros países 9 . Com mais de 30\% dos óbitos neste subgrupo etário, as doenças cardiovasculares $^{8,10}$ (sobretudo doenças isquêmicas do coração e cerebrovasculares) representam a principal causa de morte, e associam-se a fatores de risco como a hipertensão arterial e o diabetes mellitus $^{11}$, que ocorrem com maior frequência e gravidade com o aumento da idade ${ }^{12}$. No Brasil, já no ano de $2007,72 \%$ de todas as mortes foram atribuídas às doenças não transmissíveis, com distribuição heterogênea na população ${ }^{13}$.

Neste estudo, considerou-se a hipótese de que condições frequentes entre os idosos, tais como fragilidade, limitações funcionais e isolamento social elevam o risco de óbito por todas as causas, independentemente das características sociodemográficas e da presença de doenças crônicas (como a doença cardiovascular) que se associam ao óbito em todas as faixas etárias.

Dados de uma revisão sistemática com estudos de base populacional publicados entre 1990 e 2010, apontam que idosos frágeis têm taxas de sobrevivência mais baixas do que os não frágeis, com elevação de $50 \%$ do risco com base no fenótipo de fragilidade ${ }^{14}$. Estudo comparativo sobre medidas de fragilidade em relação à acurácia na predição de mortalidade revelou maior risco de óbito nos idosos frágeis, classificados pelo fenótipo de fragilidade ${ }^{15}$.

Com o envelhecimento populacional e a maior carga de doenças crônicas observa-se um aumento da perda funcional e morte prematu$\mathrm{ra}^{16}$. O estudo Epidoso que acompanhou idosos durante 10 anos, em quatro ondas de inquéritos domiciliares, detectou incidência de incapacidade funcional de $17,8 \%{ }^{17}$. A perda de autonomia para a realização de atividades instrumentais da vida diária (AIVD), que refletem tarefas de independência social ${ }^{18}$, e sua relação com a mortalidade é pouco descrita na literatura.

Dados transversais do English Longitudinal Study of Ageing (ELSA) mostram que o isolamento social, caracterizado por não ter cônjuge, ter pouco contato presencial ou por telefone com familiares e amigos e não participar de organizações sociais (clubes, grupos religiosos), foi associado ao maior risco de morte por todas as causas (hazard ratio $=1,28)^{19}$. Analisando a coorte idosa do Projeto Bambuí (2004 a 2011), Gontijo et al. ${ }^{20}$ observaram que a participação social foi independentemente associada à mortalidade, com risco de morte duas vezes maior nos idosos que não participavam de grupos sociais ou associações comunitárias.

A identificação de condições decorrentes de exposições pregressas e atuais, e cuja ocorrência também varia de acordo com as características sociodemográficas, reforça a necessidade de estimar a magnitude do risco independente de óbito nos idosos, e identificar os fatores passíveis de intervenção, de modo a subsidiar ações e estratégias que minimizem as condições preditoras do óbito. Utilizando os dados do Estudo Fibra (Fragilidade em Idosos Brasileiros) $)^{21}$ objetivou-se estimar o risco de óbito e sua relação com características sociodemográficas, doenças crônicas, fragilidade, capacidade funcional e participação social em idosos comunitários e verificar, para as variáveis de estado de saúde e participação social, o tempo mediano de ocorrência do óbito.

\section{Métodos}

Trata-se de um estudo longitudinal retrospectivo com idosos de 65 anos ou mais. Os dados foram 
obtidos da linha de base (2008-2009) e do seguimento (2016-2017) do Estudo Fibra, realizado no município de Campinas-São Paulo e em Ermelino Matarazzo, subdistrito da cidade de São Paulo.

$\mathrm{Na}$ linha de base, setores censitários foram selecionados por sorteio e os grupos foram estratificados por sexo e idade conforme a distribuição censitária da população de homens e mulheres (idade $\geq 65$ anos) dessas localidades. As localidades foram selecionadas por conveniência ${ }^{21}$, com as amostras finais contando com 900 idosos em Campinas e 384 em Ermelino Matarazzo. Para o estudo de seguimento (2016-2017), o recrutamento teve como base as listas dos endereços domiciliares registrados nos bancos de dados da linha de base. Os endereços foram percorridos pelos recrutadores para localizar os participantes e para a realização da coleta de dados no domicílio do idoso. Foram efetuadas até três tentativas de localização de cada participante. Em ambos os municípios, a confirmação do óbito e as informações sobre os falecidos foram obtidas de um familiar e/ou um conhecido do idoso. Os entrevistadores também coletavam dados do informante. Foram obtidos dados de 741 idosos (523 de Campinas e 218 de Ermelino Matarazzo), dos quais 192 (129 e 63 de Campinas e Ermelino, respectivamente) haviam falecido na ocasião do Estudo Fibra seguimento em 2016-2017 (Figura 1).

\section{Variáveis e medidas}

A variável de interesse deste estudo foi a ocorrência de óbito no período ( de falecimento do idoso, a data do óbito foi verificada com um familiar (informante) no estudo de seguimento (2016-2017). Para a análise dos fatores de risco - sociodemográficos e de saúde relacionados - foram consideradas as seguintes variáveis do banco de dados da linha de base:

- Sociodemográficas: sexo (feminino e masculino), faixa de idade (65 a 69, 70 a 74 e 75 anos e mais), escolaridade (nenhuma, 1 a 4 e 5 ou mais anos de estudo) e renda familiar mensal per capita (em quartis: até $\mathrm{R} \$ 830, \mathrm{R} \$ 831-1.200, \mathrm{R} \$$ 1.201-2.000 e acima de $\mathrm{R} \$ 2.001)$.

- Doenças crônicas: as morbidades foram avaliadas a partir de nove itens dicotômicos, baseados em autorrelato, que investigavam se algum médico havia realizado diagnóstico prévio de doença do coração, hipertensão arterial sistêmi$\mathrm{ca}$, acidente vascular cerebral/isquemia/derrame, diabetes mellitus, câncer, artrite ou reumatismo, depressão, doenças dos pulmões e osteoporose (sim ou não).

- Capacidade funcional: foi avaliada por meio de autorrelato dos idosos quanto à ajuda necessária para a execução de sete atividades instrumentais de vida diária (AIVDs): uso de telefone e de

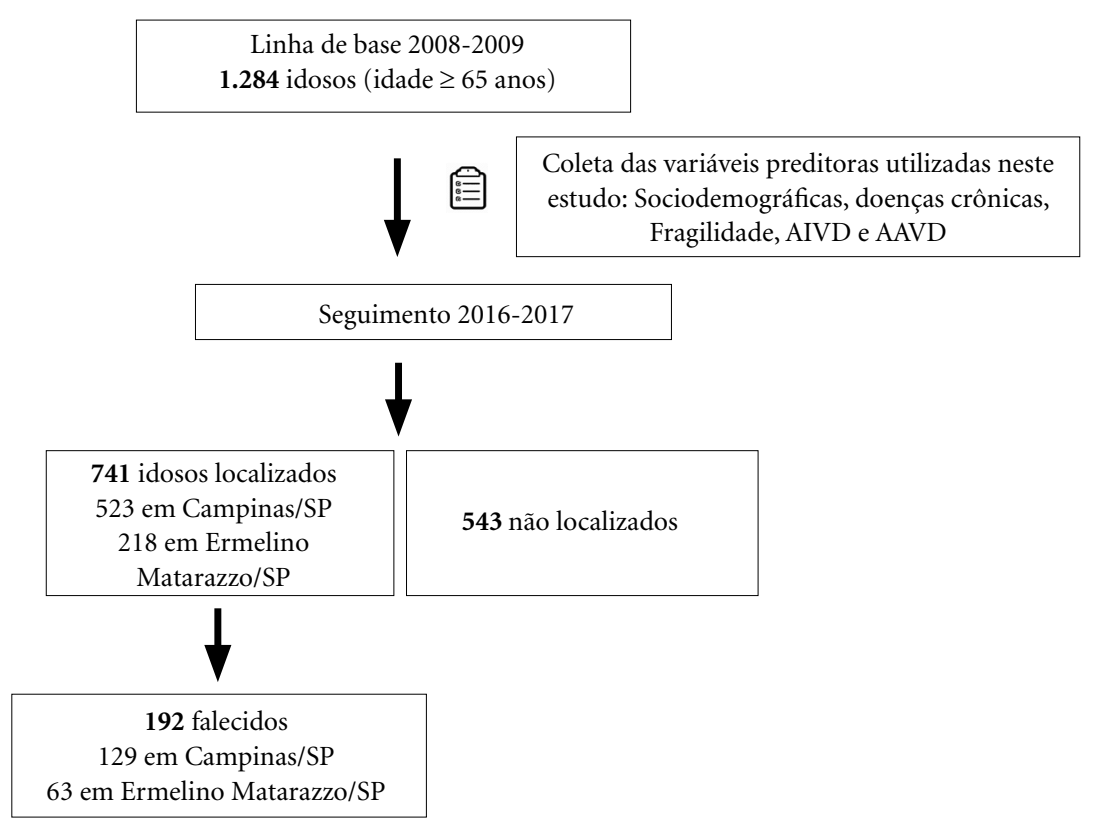

Figura 1. Fluxograma das etapas do Estudo Fibra 2008/09 e 2016/17. 
transportes, autogestão do uso de medicamentos, manejo do dinheiro, capacidade de fazer compras, de cozinhar e realizar serviços domésticos. Foram considerados dependentes os idosos que manifestaram precisar de ajuda parcial ou total para a realização de uma ou mais atividades ${ }^{22,23}$.

- Fragilidade: condição investigada por meio dos cinco critérios propostos por Fried et al. ${ }^{24}$. Classificaram-se como frágeis os idosos que apresentaram três ou mais critérios, como pré-frágeis os que pontuaram para um ou dois, e os não frágeis aqueles que não pontuaram em nenhum dos cinco critérios:

1. Perda de peso não intencional no último ano (sim ou não). Em caso de resposta positiva, perguntava-se qual a redução (em quilogramas), considerando afirmativo os idosos que relataram perda superior a $4,5 \mathrm{~kg}$ ou $5 \%$ do peso corporal.

2. Fadiga, aferida por dois itens de autorrelato obtidos do Center for Epidemiologic Studies Depression Scale (CESD), com quatro possibilidades de resposta (sempre; na maioria das vezes; poucas vezes; nunca ou raramente). Foram considerados com respostas afirmativas aqueles que responderam "sempre" ou "na maioria das vezes" para qualquer uma das duas questões.

3. Força de preensão manual, medida com dinamômetro Jamar (Lafayette Instruments, Lafayette, Indiana, Estados Unidos) colocado na mão dominante do idoso, em três tentativas, respeitando um minuto de intervalo entre elas. Foram considerados com força reduzida aqueles idosos cuja média das três aferições esteve entre os 20\% menores valores da distribuição, ajustados pelo sexo e Índice de Massa Corporal (IMC $=$ peso $[\mathrm{kg}] /$ altura $\left.^{2}[\mathrm{~m}]\right)$, conforme as faixas sugeridas pela Organização Mundial da Saúde (OMS) e descritas por Marucci e Barbosa ${ }^{25}$.

4. Velocidade da marcha, indicada pelo tempo médio, em segundos, gasto pelo idoso para percorrer por três vezes, em passo usual, no plano, uma distância de 4,6 metros, conforme as recomendações de Guralnik et al. ${ }^{26}$ e Nakano et al. ${ }^{27}$. Foram considerados com velocidade reduzida aqueles cuja média das três medidas esteve entre os 20\% maiores valores da distribuição do tempo. As médias foram ajustadas pela mediana da altura para homens e para mulheres.

5. Atividade física: corresponde à frequência semanal e à duração diária de exercício físico, esportes ativos e tarefas domésticas, com base em respostas aos itens do Minnesota Leisure Time Activity Questionnaire ${ }^{24,28,29}$. Para o cálculo do gasto calórico semanal em atividades de lazer e em atividades domésticas foi considerado o número de itens aos quais o idoso respondeu afirmativamente, multiplicado pelo número de dias na semana e pelo número de minutos por dia em que eram praticados. Em seguida, foram calculados os quintis da distribuição dessa variável para os homens e para as mulheres separadamente. Foram considerados como inativos os idosos que pontuaram entre os $20 \%$ menores valores da distribuição de gasto calórico semanal para o respectivo sexo.

- Variáveis indicativas de envolvimento social: foram consideradas quatro atividades avançadas de vida diária (AAVD), quais sejam: a realização de visitas à casa de amigos ou familiares, frequência à igreja/templo religioso para rituais religiosos ou atividades sociais ligadas à religião, participação em reuniões sociais, festas ou bailes, encontro com outras pessoas em lugares públicos, tais como restaurantes, cinemas, teatros, concertos, clubes etc. Todas essas variáveis foram categorizadas em nunca fez, parou ou ainda faz, e classificadas em sim ou não (faz ou não faz).

\section{Análise de dados}

Inicialmente foi realizada análise descritiva das características sociodemográficas e condições investigadas, a partir das medidas de frequência absoluta e relativa das variáveis categóricas, além da comparação entre proporções pelo teste QuiQuadrado de Pearson, com nível de significância de $5 \%$, para o conjunto de idosos localizados ou não encontrados (perdas), no estudo de seguimento.

Em seguida, os idosos para os quais foi possível obter dados no seguimento foram classificados segundo a ocorrência de óbito (sim ou não) no período, e foram verificados o número absoluto e a incidência acumulada (\%) de acordo com as variáveis selecionadas. Também foram estimadas as razões de incidência (risco relativo) de morte, brutas e ajustadas, por sexo, idade e escolaridade, e respectivos intervalos de confiança de $95 \%$.

A análise múltipla de regressão de Poisson obedeceu ao seguinte modelo hierárquico: na primeira etapa foram incluídas as características sociodemográficas associadas à mortalidade. $\mathrm{Na}$ segunda, além das variáveis pertencentes ao primeiro bloco e que mantiveram significância após ajuste pelas demais variáveis do mesmo nível hierárquico, foram acrescentadas as variáveis doenças crônicas, fragilidade, capacidade funcional e os indicadores de envolvimento social. Foram incluídas nos modelos as variáveis que apresen- 
taram associação significante com a mortalidade na análise simples $(\mathrm{p}<0,20)$. No modelo final, restaram aquelas que apresentaram um valor de $\mathrm{p}<0,05$, quando ajustadas pelas variáveis do nível superior e do mesmo nível hierárquico.

Para a análise do tempo até a ocorrência do óbito, considerando-se as variáveis que apresentaram associação independente com a mortalidade no período, foi criada a variável tempo (em meses) a partir das datas da $1^{\text {a }}$ entrevista e do óbito. Pelo método de Kaplan Meier, foram estimados os tempos medianos de sobrevivência, bem como as curvas para cada variável. Verificou-se a igualdade das distribuições de sobrevivência, para os diferentes níveis das variáveis consideradas, pelo teste Log-rank (Mantel-Cox), com um nível de significância de 5\%. Na análise dos dados foram utilizados os programas Stata 15.1 e SPSS versão 21 .

\section{Aspectos éticos}

Os projetos foram encaminhados e aprovados pelo Comitê de Ética da Universidade Estadual de Campinas. Todos os participantes, informados sobre os objetivos da pesquisa, procedimentos e seus direitos, assinaram o Termo de Consentimento Livre e Esclarecido.

\section{Resultados}

Neste estudo foram analisadas informações de 741 idosos que participaram da linha de base em 2008-2009, com média de idade de 72,6 anos $(d p=5,8)$ e $67,3 \%$ de mulheres. Na Tabela $1 \mathrm{ob}-$ serva-se que somente as variáveis faixa etária e indicador de envolvimento social apresentaram diferenças entre as proporções $(\mathrm{p}<0,05)$ para o conjunto dos idosos localizados ou não no estudo de seguimento. No que se refere à comparação entre os idosos localizados $(n=549)$ e não localizados por motivo de falecimento $(n=192)$ quase todas as variáveis apresentaram diferenças significativas, exceto câncer, derrame, doença pulmonar, diabetes mellitus e hipertensão (dados não apresentados em Tabela).

Observou-se maior risco de morte entre os idosos mais velhos ( $R R=1,85$; IC95\%: 1,30-2,63), com doença do coração ( $R R=1,58$; IC95\%: 1,112,26), pré-frágeis $(\mathrm{RR}=1,53$; IC95\%: 1,06-2,20) ou frágeis $(R R=1,74$; IC95\%: 1,09-2,79) e naqueles que relataram dependência para a realização das atividades instrumentais da vida diária (AIVD) (RR=1,61; IC95\%: 1,13-2,29). Nas mu- lheres o risco foi $28 \%$ menor, assim como naqueles com maior escolaridade ( $\mathrm{RR}=0,64$; IC95\%: $0,41-0,98)$ e naqueles que relataram três ou mais atividades avançadas de vida diária (Tabela 2).

Os resultados da análise de regressão hierárquica são apresentados na Tabela 3. A incidência de óbito foi significativamente menor nas mulheres $(\mathrm{RP}=0,64$; IC95\%: 0,43-0,95), naqueles com maior renda familiar ( $\mathrm{RP}=0,51$; IC95\%: 0,27 $0,96)$ e nos que relataram a realização de três ou mais atividades relacionadas à inserção social $(\mathrm{RP}=0,53$, IC95\%: 0,32-0,87). Nos mais idosos, naqueles que relataram doença do coração e nos que apresentaram dependência para realização das atividades instrumentais da vida diária, a incidência de óbito foi maior $(\mathrm{p}<0,05)$.

O tempo mediano geral de ocorrência do óbito entre os participantes que relataram ter doença do coração foi de 62 meses (IC95\%: 54,5$69,5)$; para aqueles com limitações na realização de atividades instrumentais da vida diária, assim como para aqueles que relataram realizar menos do que três AAVD foi de 60 meses (Figura 2).

\section{Discussão}

Os principais achados deste estudo foram verificar que além do sexo masculino, maior idade e menor renda, a dificuldade para realização de atividade instrumental da vida diária, o menor envolvimento social e a presença de doença do coração elevaram, de forma independente, o risco de óbito nos idosos. O contexto de vida em que se insere a maioria dos idosos brasileiros, sujeitos aos efeitos cumulativos decorrentes de baixo nível econômico, baixa escolaridade e ausência de suporte social, exige a incorporação de uma abordagem de cuidado que considere várias dimensões ${ }^{4,5,7,21}$.

A sobremortalidade masculina tem sido observada em todas as idades e grupos de causas no Brasil ${ }^{30}$. A maior expectativa de vida das mulheres em relação aos homens resulta, em grande parte, de diferenças nas atividades de trabalho, estilo de vida e comportamentos relacionados à saúde ${ }^{30-32}$. Embora sejam observadas variações nas diferentes regiões do mundo, proporcionalmente as mulheres ultrapassam a metade da população idosa ${ }^{33}$.

As desigualdades socioeconômicas e aumento da concentração de renda e sua relação com a mortalidade são amplamente descritas na literatura $^{34,35}$. O Brasil é o $10^{\circ}$ país do mundo em concentração da renda e o primeiro quanto ao grau 
Tabela 1. Características dos idosos ( $\geq 65$ anos) que permaneceram ou não no seguimento. Estudo Fibra 2008/09 e 2016/17.

\begin{tabular}{|c|c|c|c|c|c|}
\hline \multirow{2}{*}{ Variáveis } & \multicolumn{2}{|c|}{ Seguimento } & \multicolumn{2}{|c|}{ Perda de seguimento } & \multirow{2}{*}{$\begin{array}{l}\text { Valor } \\
\text { de } p^{*}\end{array}$} \\
\hline & $n=751$ & $\%$ & $\mathrm{n}=543$ & $\%$ & \\
\hline \multicolumn{6}{|l|}{ Sexo } \\
\hline Masculino & 242 & 32,7 & 160 & 29,5 & 0,223 \\
\hline Feminino & 499 & 67,3 & 383 & 70,5 & \\
\hline \multicolumn{6}{|l|}{ Faixa etária (em anos) } \\
\hline 65 a 69 & 241 & 32,5 & 214 & 39,4 & 0,011 \\
\hline 70 a 74 & 234 & 31,6 & 173 & 31,9 & \\
\hline 75 ou mais & 266 & 35,9 & 156 & 28,7 & \\
\hline \multicolumn{6}{|l|}{ Escolaridade (em anos) } \\
\hline 0 & 140 & 18,9 & 93 & 17,2 & 0,069 \\
\hline 1 a 4 & 430 & 58,1 & 293 & 54,2 & \\
\hline 5 ou mais & 170 & 23,0 & 155 & 28,6 & \\
\hline \multicolumn{6}{|l|}{ Renda familiar (em quartis)\# } \\
\hline $1^{\circ}$ quartil & 188 & 29,6 & 121 & 25,8 & 0,208 \\
\hline $2^{\circ}$ quartil & 138 & 21,7 & 119 & 25,4 & \\
\hline $3^{\circ}$ quartil & 177 & 27,9 & 118 & 25,2 & \\
\hline $4^{\circ}$ quartil & 132 & 20,8 & 110 & 23,5 & \\
\hline \multicolumn{6}{|l|}{ Diagnóstico médico de doenças } \\
\hline Hipertensão arterial & 381 & 64,7 & 257 & 63,9 & 0,807 \\
\hline Artrite/artrose/reumatismo & 237 & 40,2 & 170 & 42,3 & 0,519 \\
\hline Doença do coração & 149 & 25,3 & 101 & 25,1 & 0,921 \\
\hline Diabetes mellitus & 135 & 22,9 & 90 & 22,4 & 0,844 \\
\hline Doenças pulmonares & 68 & 11,6 & 37 & 9,2 & 0,236 \\
\hline Câncer & 57 & 9,7 & 28 & 7,0 & 0,134 \\
\hline Derrame/AVC & 52 & 8,8 & 28 & 6,9 & 0,285 \\
\hline \multicolumn{6}{|l|}{ Fragilidade } \\
\hline Não frágil & 223 & 30,1 & 163 & 30,0 & 0,857 \\
\hline Pré-frágil & 429 & 57,9 & 320 & 58,9 & \\
\hline Frágil & 89 & 12,0 & 60 & 11,1 & \\
\hline \multicolumn{6}{|l|}{ Desempenho de atividades de vida diária } \\
\hline Dependente nas AIVD (parcial/total) & 176 & 30,2 & 120 & 29,7 & 0,856 \\
\hline \multicolumn{6}{|c|}{ Indicador de envolvimento social (número de AAVD) } \\
\hline 0 a 1 & 140 & 24,1 & 79 & 19,7 & 0,005 \\
\hline 2 & 196 & 33,7 & 111 & 27,7 & \\
\hline 3 ou mais & 245 & 42,2 & 211 & 52,6 & \\
\hline
\end{tabular}

*Valor de $\mathrm{p}$ do Teste Qui-quadrado de Pearson: em negrito $\mathrm{p}<0,05$. \#Valores de renda familiar: Quartil 1 (até $\mathrm{R} \$ 830,00)$; Quartil 2 (R\$ 831,00-1.200,00); Quartil 3 (R\$ 1.201,00-2.000,00); Quartil 4 (acima de R\$2.001,00). AIVD: Atividades instrumentais de vida diária. AAVD: Atividades avançadas de vida diária.

Fonte: Elaborado pelas autoras.

de concentração da renda no centil mais rico da população ${ }^{36}$, com impactos negativos para a saúde, particularmente dos idosos ${ }^{37}$.

As atividades avançadas da vida diária (AAVD) compreendem tarefas recreativas, produtivas e sociais de maior complexidade na avaliação funcional do idoso ${ }^{38}$. Neste estudo observou-se menor risco de mortalidade entre os indivíduos que relataram alguma inserção social medida pelo número de AAVD realizadas. Para além da prevenção de doenças e limitações delas decorrentes, e de outros determinantes diretamente relacionados às condições de vida e saúde dos idosos, os marcos conceituais no campo do envelhecimento têm buscado contemplar estratégias que garantam a continuidade de participação dos idosos em diversas atividades ${ }^{4}$. Deste modo, o conceito multidimensional de Envelhe- 
Tabela 2. Incidência acumulada e risco relativo ajustado de mortalidade em idosos ( $\geq 65$ anos), segundo características sociodemográficas, doenças crônicas, fragilidade e desempenho nas atividades instrumentais de vida diária. Estudo Fibra 2008/09 e 2016/17.

\begin{tabular}{|c|c|c|c|c|}
\hline Variáveis & $\begin{array}{l}\text { Número de } \\
\text { mortes }\end{array}$ & $\begin{array}{c}\text { Incidência de } \\
\text { mortalidade \% }\end{array}$ & $\begin{array}{l}\text { RR bruta } \\
\text { (IC95\%) }\end{array}$ & $\begin{array}{l}\text { RR ajustado } \\
\text { (IC95\%) }\end{array}$ \\
\hline Sexo & & $0,011^{\star}$ & & \\
\hline Masculino & 77 & 31,8 & 1 & 1 \\
\hline Feminino & 115 & 23,0 & $0,72(0,54-0,96)$ & $0,72(0,54-0,96)$ \\
\hline Faixa etária (em anos) & & $<0,001^{\star}$ & & \\
\hline 65 a 69 & 46 & 19,1 & 1 & 1 \\
\hline 70 a 74 & 45 & 19,2 & $1,00(0,67-1,52)$ & $0,96(0,63-1,44)$ \\
\hline 75 ou mais & 101 & 38,0 & $1,99(1,40-2,82)$ & $1,85(1,30-2,63)$ \\
\hline Escolaridade (em anos) & & $0,002^{*}$ & & \\
\hline 0 & 52 & 37,1 & 1 & 1 \\
\hline 1 a 4 & 105 & 24,4 & $0,66(0,47-0,92)$ & $0,72(0,52-1,01)$ \\
\hline 5 ou mais & 35 & 20,6 & $0,55(0,36-0,85)$ & $0,64(0,41-0,98)$ \\
\hline Renda familiar (em quartis)\# & & $0,020^{*}$ & & \\
\hline $1^{\circ}$ quartil & 57 & 30,3 & 1 & 1 \\
\hline $2^{\circ}$ quartil & 32 & 23,2 & $0,76(0,50-1,18)$ & $0,81(0,52-1,25)$ \\
\hline $3^{\circ}$ quartil & 44 & 24,9 & $0,82(0,55-1,21)$ & $0,93(0,62-1,40)$ \\
\hline $4^{\mathrm{o}}$ quartil & 20 & 15,1 & $0,50(0,30-0,83)$ & $0,60(0,35-1,04)$ \\
\hline \multicolumn{5}{|l|}{ Diagnóstico médico de doenças } \\
\hline \multirow[t]{2}{*}{ Hipertensão arterial } & & $0,996^{\star}$ & & \\
\hline & 88 & 23,1 & $1,00(0,70-1,42)$ & $1,07(0,75-1,52)$ \\
\hline \multirow[t]{2}{*}{ Artrite/artrose/reumatismo } & & $0,006^{*}$ & & \\
\hline & 41 & 17,3 & $0,64(0,44-0,92)$ & $0,71(0,49-1,04)$ \\
\hline \multirow[t]{2}{*}{ Doença do coração } & & $0,008^{*}$ & & \\
\hline & 46 & 30,9 & $1,52(1,07-2,17)$ & $1,58(1,11-2,26)$ \\
\hline \multirow[t]{2}{*}{ Diabetes mellitus } & & $0,373^{\star}$ & & \\
\hline & 35 & 25,9 & $1,16(0,79-1,71)$ & $1,16(0,79-1,71)$ \\
\hline \multirow[t]{2}{*}{ Doenças pulmonares } & & $0,099^{*}$ & & \\
\hline & 21 & 30,9 & $1,41(0,88-2,24)$ & $1,37(0,86-2,19)$ \\
\hline \multirow[t]{2}{*}{ Câncer } & & $0,957^{\star}$ & & \\
\hline & 13 & 22,8 & $0,99(0,56-1,75)$ & $1,00(0,56-1,78)$ \\
\hline \multirow[t]{2}{*}{ Derrame/AVC } & & $0,998^{*}$ & & \\
\hline & 12 & 23,1 & $1,00(0,55-1,81)$ & $0,95(0,52-1,72)$ \\
\hline Fragilidade & & $<0,001^{\star}$ & & \\
\hline Não frágil & 39 & 17,5 & 1 & 1 \\
\hline Pré-frágil & 119 & 27,7 & $1,59(1,10-2,28)$ & $1,53(1,06-2,20)$ \\
\hline Frágil & 34 & 38,2 & $2,18(1,38-3,46)$ & $1,74(1,09-2,79)$ \\
\hline Atividades instrumentais de vida diária & & $<\mathbf{0 , 0 0 1 ^ { \star }}$ & & \\
\hline Independente & 74 & 18,2 & 1 & 1 \\
\hline Dependente (parcial ou total) & 60 & 34,1 & $1,87(1,33-2,63)$ & $1,61(1,13-2,29)$ \\
\hline Indicador de envolvimento social & & $<0,001^{\star}$ & & \\
\hline 0 a 1 & 45 & 33,8 & 1 & 1 \\
\hline 2 & 52 & 39,1 & $0,82(0,55-1,23)$ & $0,83(0,56-1,24)$ \\
\hline 3 ou 4 & 36 & 27,1 & $0,46(0,29-0,71)$ & $0,51(0,33-0,79)$ \\
\hline
\end{tabular}

cimento Ativo compreende não somente a participação econômica dos idosos, mas também outras formas não remuneradas, como o envolvimento em atividades sociais, culturais, intelec- 
Tabela 3. Modelo múltiplo de regressão hierárquica de Poisson e variáveis associadas à mortalidade em idosos ( $\geq 65$ anos). Estudo Fibra 2008/09 e 2016/17.

\begin{tabular}{|c|c|c|c|c|}
\hline \multirow[b]{2}{*}{ Variáveis } & \multirow{2}{*}{$\begin{array}{c}1^{\text {a }} \text { etapa }^{\star} \\
\text { RR ajustado } \\
(\text { IC95\%) }\end{array}$} & \multirow{2}{*}{$\begin{array}{c}\text { Valor } \\
\text { de } p\end{array}$} & \multirow{2}{*}{$\begin{array}{c}2^{\mathrm{a}} \text { etapa }^{\star *} \\
\text { RR ajustado } \\
\text { (IC95\%) }\end{array}$} & \multirow{2}{*}{$\begin{array}{c}\text { Valor } \\
\text { de p }\end{array}$} \\
\hline & & & & \\
\hline \multicolumn{5}{|l|}{ Sexo } \\
\hline Masculino & 1 & & 1 & \\
\hline Feminino & $0,69(0,50-0,96)$ & 0,028 & $0,64(0,43-0,95)$ & 0,027 \\
\hline \multicolumn{5}{|l|}{ Faixa etária (em anos) } \\
\hline 65 a 69 & 1 & & 1 & \\
\hline 70 a 74 & $1,08(0,69-1,70)$ & 0,729 & $1,01(0,59-1,74)$ & 0,958 \\
\hline 75 ou mais & $2,06(1,39-3,06)$ & $<0,001$ & $1,94(1,20-3,12)$ & 0,007 \\
\hline \multicolumn{5}{|l|}{ Renda familiar (em quartis)\# } \\
\hline $1^{\circ}$ quartil & 1 & & 1 & \\
\hline $2^{\circ}$ quartil & $0,76(0,49-1,18)$ & 0,222 & $0,88(0,53-1,46)$ & 0,620 \\
\hline $3^{\circ}$ quartil & $0,84(0,57-1,26)$ & 0,411 & $0,87(0,52-1,45)$ & 0,598 \\
\hline $4^{\circ}$ quartil & $0,50(0,30-0,83)$ & 0,007 & $0,51(0,27-0,96)$ & 0,037 \\
\hline \multicolumn{5}{|l|}{ Doença do coração } \\
\hline Não & & & 1 & \\
\hline $\operatorname{Sim}$ & & & $1,55(1,03-2,34)$ & 0,036 \\
\hline \multicolumn{5}{|c|}{ Atividades instrumentais de vida diária } \\
\hline Independente & & & 1 & \\
\hline Dependente (parcial ou total) & & & $1,66(1,11-2,47)$ & 0,013 \\
\hline \multicolumn{5}{|c|}{ Indicador de envolvimento social (número de AAVD) } \\
\hline 0 a 1 & & & 1 & \\
\hline 2 & & & $0,91(0,58-1,43)$ & 0,690 \\
\hline 3 ou mais & & & $0,53(0,32-0,87)$ & 0,013 \\
\hline
\end{tabular}

Fonte: Elaborado pelas autoras.

tuais, físicas e políticas ${ }^{39,40}$. Em adultos brasileiros mais velhos, além da idade e da escolaridade, também a mobilidade, a sociabilidade e o suporte instrumental e emocional associaram-se à qualidade de vida percebida ${ }^{41}$.

A ideia de que a velhice é dominada pela doença nem sempre se mostra como realidade, pois, mesmo existindo perdas, tanto no nível biológico como econômico, social e psicológico, a manutenção das atividades e do engajamento social e familiar favorece o envelhecimento saudável ${ }^{42}$ (p.1765).

$\mathrm{O}$ risco independente de óbito por doença do coração (como angina, infarto do miocárdio ou ataque cardíaco etc.) corrobora o grande impacto das doenças cardiovasculares na morbidade e mortalidade da população, principalmente representadas pelas doenças isquêmicas do coração (DIC) e doenças cerebrovasculares (DCbV) ${ }^{13,43}$.
Ressalta-se que, nos idosos, para além dos custos elevados de internação e de seguimento ambulatorial, essas doenças continuam sendo a principal causa de morte $\mathrm{e}^{43,44}$.

Fatores de risco para doenças cardiovasculares incluem a hipertensão arterial e diabetes mellitus com elevadas prevalências entre os ido$\operatorname{sos}^{45-47}$, dislipidemias, inatividade física, sobrepeso/obesidade e alimentação inadequada, além do tabagismo e consumo abusivo de álcool entre outros fatores ${ }^{48}$ menos frequentes nesta faixa etária. Parte das mortes por doenças do coração entre os idosos pode ser caracterizada como evitável $^{49}$ se considerarmos que este evento decorre de efeitos que poderiam ser controlados, seja por meio do diagnóstico e tratamento precoces, de ações de prevenção de complicações clínicas de condições de base pré-existentes, bem como 
A

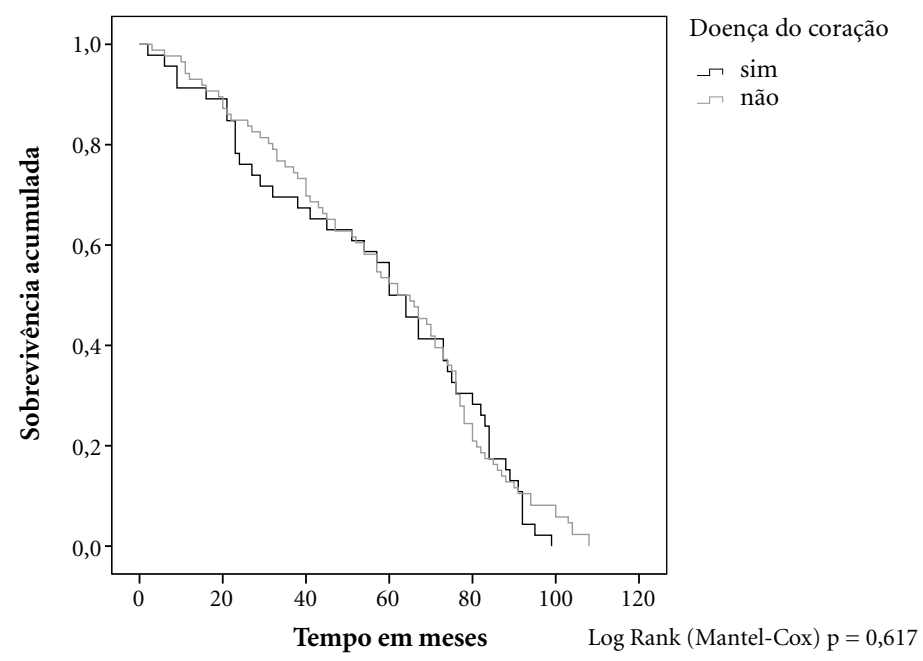

B

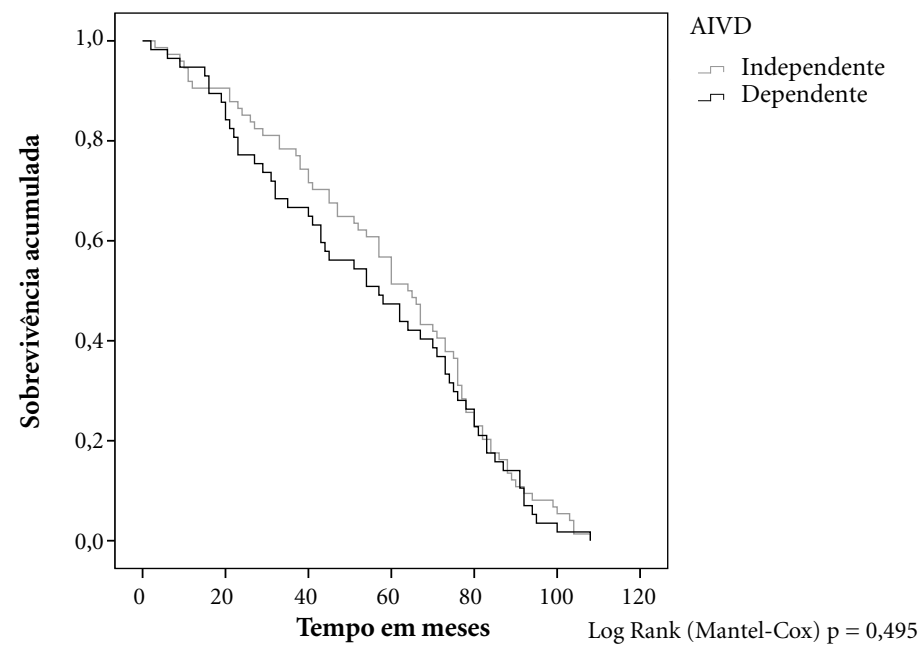

C

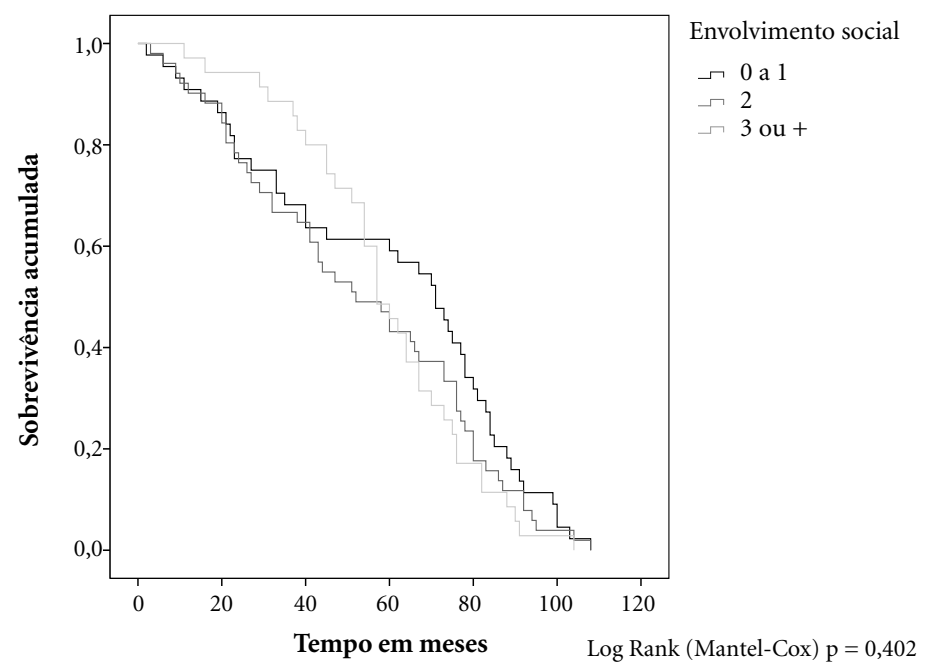

Figura 2. Curvas de sobrevida Kaplan-Meier segundo doença do coração (A), AIVD (B) e indicador de envolvimento social (C).

Fonte: Elaborado pelas autoras. 
do uso de tecnologias de forma mais oportuna, principalmente entre os economicamente menos favorecidos ${ }^{34,35,50}$.

Quanto ao tempo decorrido à incidência dos óbitos, não houve diferença estatisticamente significativa para as variáveis associadas ao risco de morte. O tempo mediano até o óbito nos idosos foi de cerca de 60 meses ( 5 anos). Deve-se considerar que os participantes do Estudo Fibra constituem uma amostra de idosos sem sinais evidentes de demência na linha de base e que compareceu em espaços públicos para a coleta de dados, o que pode ter inserido um viés de seleção ao estudo possibilitando a maior participação de indivíduos com melhor condição física, emocional e cognitiva ${ }^{21}$. Particularmente para o desfecho estudado (óbito), tanto as melhores condições na linha de base, quanto o tempo de segmento (cerca de 8 anos), podem ter influenciado na magnitude do risco e nas diferenças não observadas no tempo mediano de ocorrência do óbito.

Quanto à coorte de idosos, em relação a todas as variáveis consideradas no presente estudo, não houve perda diferencial de seguimento, exceto para a idade que se associa à realização de ati-

\section{Colaboradores}

PMSB Francisco orientou a proposta do estudo, análise, interpretação dos dados, redação do texto e revisão crítica final. D Assumpção realizou a análise, interpretação dos dados, redação do texto e revisão crítica final. FSA Borim contribuiu com a revisão da literatura e revisão crítica da versão final do artigo. MS Yassuda participou da coleta de dados e revisão crítica da versão final do artigo. AL Neri realizou a concepção do Estudo Fibra, coleta de dados e revisão crítica da versão final. vidades relacionadas ao envelhecimento ativo ${ }^{51}$. Naqueles localizados o percentual com idade igual ou superior a 75 anos foi significativamente maior, o que poderia compensar, parcialmente, $o$ viés de seleção, dado que a idade é sabidamente preditora independente de óbito principalmente nos idosos. Embora o envelhecimento e a doença não constituam fatores intimamente dependentes, existe maior risco de adoecimento nesta fase da vida.

Neste estudo, além da idade e do sexo masculino, a pior condição socioeconômica e de mobilidade elevaram o risco de morte dos idosos no período. Entre as condições de saúde investigadas, somente a doença do coração esteve associada ao óbito após ajuste pelas demais variáveis. Ressalta-se que a sociabilidade esteve inversamente associada à ocorrência de óbito. Os resultados nos permitem refletir sobre a complexidade do processo saúde-doença-cuidado na população idosa, ao mesmo tempo em que apontam para singularidades inerentes aos indivíduos no que tange à dimensão social que exerce efeitos diversos sobre a morbidade e mortalidade, e que não podem ser diretamente mensurados.

\section{Agradecimentos}

À Coordenação de Aperfeiçoamento de Pessoal de Nível Superior - Brasil (CAPES) - Código de Financiamento 001, à Fundação de Amparo à Pesquisa do Estado de São Paulo (FAPESP), e ao Conselho Nacional de Desenvolvimento Científico e Tecnológico $(\mathrm{CNPq})$ pelo financiamento à pesquisa. 


\section{Referências}

1. United Nations (UN). Department of Economic and Social Affairs, Population Division. World Population Prospects: the 2017 revision [Internet]. New York: UN; 2017 [acessado 2018 jul 17]. Disponível em: http:// esa.un.org/unpd/wpp/.

2. Miranda GMD, Mendes ACG, Silva ALA. Population aging in Brazil: current and future social challenges and consequences. Rev Bras Geriatr Gerontol 2016; 19(3):507-519.

3. Barros MBA, Francisco PMSB, Zanchetta LM, Cesar CLG. Tendências das desigualdades sociais e demográficas na prevalência de doenças crônicas no Brasil, PNAD: 2003-2008. Cien Saude Colet 2011; 16(9):3755-3768.

4. Barros MBA, Goldbaum M. Desafios do envelhecimento em contexto de desigualdade social. Rev Saude Publica 2018; 52(Supl. 2):1s

5. Giacomin KC, Duarte YAD, Camarano AA, Nunes DP, Fernandes D. Cuidado e limitações funcionais em atividades cotidianas - ELSI-Brasil. Rev Saude Publica 2018; 52(Supl. 2):9s.

6. Stopa SR, Malta DM, Monteiro CN, Szwarcwald CL, Goldbaum M, Cesar CLG. Acesso e uso de serviços de saúde pela população brasileira, Pesquisa Nacional de Saúde 2013. Rev Saude Publica 2017; 51(Supl. 1):3s.

7. Veras RP, Caldas CP, Cordeiro HA. Modelos de atenção à saúde do idoso: repensando o sentido da prevenção. Physis 2013; 23(4):1189-1213.

8. Brasil. Ministério da Saúde (MS). Informações de Saúde (Tabnet). Estatísticas vitais [Internet]. 2020 [acessado 2020 mar 15]. Disponível em: http://www2.datasus.gov.br/DATASUS/index.php?area $=0205$.

9. GBD 2017 Mortality Collaborators. Global, regional, and national age-sex-specific mortality and life expectancy, 1950-2017: a systematic analysis for the Global Burden of Disease Study 2017. Lancet 2018; 392(10159):1684-1735.

10. Oliveira TC, Medeiros WR, Lima KC. Diferenciais de mortalidade por causas nas faixas etárias limítrofes de idosos. Rev Bras Geriatr Gerontol 2015; 18(1):85-94.

11. Brasil. Ministério da Saúde (MS). Secretaria de Vigilância em Saúde. Departamento de Análise de Situação de Saúde. Plano de ações estratégicas para o enfrentamento das doenças crônicas não transmissíveis (DCNT) no Brasil 2011-2022. Brasília: MS; 2011.

12. World Health Organization (WHO). Cardiovascular disease [Internet]. 2016 [acessado 2020 abr 22]. Disponível em: http://www.who.int/cardiovascular_diseases/en/.

13. Schmidt MI, Duncan BB, Silva GA, Menezes AM, Monteiro CA, Barreto SM, Chor D, Menezes PR. Chronic non-communicable diseases in Brazil: burden and current challenges. Lancet 2011; 377(9781):19491961.

14. Shamliyana T, Talley KMC, Ramakrishnan R, Kanea RL. Association of frailty with survival: A systematic literature review. Ageing Res Rev 2013; 12:719-736.

15. Pereira AA, Borim FSA, Aprahamian I, Neri AL. Comparison of two models of frailty for the prediction of mortality in brazilian community-dwelling older adults: the fibra study. J Nutr Health Aging 2019; 23(10):1004-1010.
16. Marinho F, Passos VMA, França EB. Novo século, novos desafios: mudança no perfil da carga de doença no Brasil de 1990 a 2010. Epidemiol Serv Saude 2016; 25(4):713-724.

17. d'Orsi E, Xavier AJ, Ramos LR. Trabalho, suporte social e lazer protegem idosos da perda funcional: Estudo Epidoso. Rev Saude Publica 2011; 45(4):685-692.

18. Guralnik JM, Fried LP, Salive ME. Disability as a Public Health Outcome in the Aging Population. Annu Rev Public Health 1996; 17:25-46.

19. Smith SG, Jackson SE, Kobayashi LC, Steptoe A. Social isolation, health literacy, and mortality risk: findings from the English Longitudinal Study of Ageing. Health Psychol 2018; 37(2):160-169.

20. Gontijo CF, Firmo JOA, Lima-Costa MF, Loyola Filho AI. Um estudo longitudinal da associação do capital social e mortalidade entre idosos brasileiros residentes em comunidade. Cad Saude Publica 2019; 35(2):e00056418.

21. Neri AL, Yassuda MS, Araújo LF, Eulálio MC, Cabral BE, Siqueira MEC, Santos GA, Moura JGA. Metodologia e perfil sociodemográfico, cognitivo e de fragilidade de idosos comunitários de sete cidades brasileiras: Estudo FIBRA. Cad Saude Publica 2013; 29(4):778-792.

22. Lawton MP, Brody EM. Assesment of older people: self-maintaining and instrumental activities of daily living. Gerontologist 1969; 9(3):179-186.

23. Santos RL, Virtuoso Júnior JS. Confiabilidade da versão brasileira da escala de atividades instrumentais da vida diária. RBPS 2008; 21(4):290-296.

24. Fried LP, Tangen CM, Walston J, Newman AB, Hirsch C, Gottdiener J, Seeman T, Tracy R, Kop WJ, Burke G, McBurnie MA, Cardiovascular Health Study Collaborative Research Group. Frailty in older adults: evidence for a phenotype. J Gerontol A Biol Sci Med Sci 2001; 56(3):M146-M156.

25. Marucci M, Barbosa A. Estado nutricional e capacidade física. In: Lebrão ML, Duarte YAO, organizadoras SABE - Saúde, Bem-estar e Envelhecimento: o Projeto SABE no município de São Paulo: uma abordagem inicial. Brasília: OPAS; 2003. p. 93-117.

26. Guralnik JM, Simonsick EM, Ferrucci L, Glynn RJ, Berkman LF, Blazer DG, Scherr PA, Wallace RB. A short physical performance battery assessing lower extremity function: association with self-reported disability and prediction of mortality and nursing home admission. J Gerontol Med Sci 1994; 49(2):M85-M94.

27. Nakano MM. Adaptacão cultural do instrumento Short Physical Performance Battery - SPPB: adaptação cultural e estudo da confiabilidade [dissertação]. Campinas: Universidade Estadual de Campinas; 2007.

28. Lustosa L, Pereira D, Dias R, Britto R, Pereira L. Tradução, adaptação transcultural e análise das propriedades psicométricas do Questionário Minnesota de Atividades Físicas e de Lazer. Belo Horizonte: Universidade Federal de Minas Gerais; 2010

29. Taylor HL, Jacobs Jr DR, Schucker B, Knudsen J, Leon AS, Debacker G. A questionnaire for the assessment of leisure time physical activities. J Chron Dis 1978; 31(12):741-755.

30. Laurenti R, Mello Jorge MHP, Gotlieb SLD. Perfil epidemiológico da morbi-mortalidade masculina. Cien Saude Colet 2005; 10(1):35-46. 
31. Sundberg L, Agahi N, Fritzell J, Fors S. Why is the gender gap in life expectancy decreasing? The impact of age- and cause-specific mortality in Sweden 19972014. Int J Public Health 2018; 63(6):673-681.

32. Szwarcwald CL, Montilla DER, Marques AP, Damacena GN, Almeida WS, Malta DC. Desigualdades na esperança de vida saudável por Unidades da Federação. Rev Saude Publica 2017; 51(Supl. 1):7s.

33. United Nations (UN). Department of Economic and Social Affairs. The World's Women 2010: Trends and Statistics [Internet]. New York: UN; 2010 [acessado 2018 jul 17]. Disponível em: https://unstats.un.org/ unsd/demographic-social/products/worldswomen.

34. Hoffmann R, Hu Y, Gelder R, Menville G, Bopp M, Mackenbach JP. The impact of increasing income inequalities on education inequalities in mortality: an analysis of six European countries. Int J Equity Health 2016; 15:103.

35. Mackenbach JP, Kulhánová I, Menvielle G, Bopp M, Borrell C, Costa G, Deboosere P, Esnaola S, Kalediene R, Kovacs K, Leinsalu M, Martikainen P, Regidor E, Rodriguez-Sanz M, Strand BH, Hoffmann R, Eikemo TA, Östergren O, Lundberg O, Eurothine and EUROGBD-SE consortiums. Trends in inequalities in premature mortality: a study of 3.2 million deaths in 13 European countries. J Epidemiol Community Health 2015; 69(3):207-217.

36. Georges R, Maia K. A distância que nos une: um retrato das desigualdades brasileiras: relatório [Internet]. São Paulo: OXFAM; 2017 [acessado 2018 abr 20]. Disponível em: https://oxfam.org.br/um-retrato-das-desigualdades-brasileiras/a-distancia-que-nos-une/.

37. Danielewicz AL, d'Orsi E, Boing AF. Renda contextual e incidência de incapacidade: resultados da Coorte EpiFloripa Idoso. Rev Saude Publica 2019; 53:11.

38. Dias EG, Duarte YAO, Almeida HM, Lebrão ML. Caracterização das atividades avançadas de vida diária (AAVDS): um estudo de revisão. Rev Ter Ocup Univ São Paulo 2011; 22(1):45-51.

39. Centro Internacional de Longevidade Brasil (ILC -Brasil). Envelhecimento ativo: um marco político em resposta à revolução da longevidade. Rio de Janeiro: ILC-Brasil; 2015.

40. World Health Organization (WHO). Envelhecimento ativo: uma política de saúde. Brasília: OPAS; 2005.

41. Neri AL, Borim FSA, Fontes AP, Rabello DF, Cachioni M, Batistoni SST, Yassuda MS, Souza Junior PRB, Andrade FB, Lima-Costa MF. Fatores associados à qualidade de vida percebida em adultos mais velhos: ELSI-Brasil. Rev Saude Publica 2018; 52(Supl. 2):16s.

42. Ciosak SI, Braz E, Costa MFBNA, Gonçalves N, Nakano R, Rodrigues J, Alencar RA, Rocha ACAL. Senescência e senilidade: novo paradigma na Atenção Básica de Saúde. Rev Esc Enferm USP 2011; 45(n. esp. 2):1763-1768.

43. Lotufo PA. Trends in cardiovascular diseases and heart disease death rates among adults aged 45-64: Brazil, 2000-2017. Sao Paulo Med J 2019; 137(3):213-215.
44. Piuvezam G, Medeiros WR, Costa AV, Emerenciano FF, Santos RC, Seabra DS. Mortalidade em Idosos por Doenças Cardiovasculares: Análise Comparativa de Dois Quinquênios. Arq Bras Cardiol 2015; 105(4):371380.

45. Francisco PMSB, Borim FS, Segri NJ, Malta DC. Prevalência simultânea de hipertensão e diabetes em idosos brasileiros: desigualdades individuais e contextuais. Cien Saude Colet 2018; 23(11):3829-3840.

46. Andrade SSA, Stopa SR, Brito AS, Chueri PS, Szwarcwald CL, Malta DC. Prevalência de hipertensão arterial autorreferida na população brasileira: análise da Pesquisa Nacional de Saúde, 2013. Epidemiol Serv Saude 2015; 24(2):297-304.

47. Iser BPM, Stopa SR, Chueiri PS, Szwarcwald CL, Malta DC, Monteiro HOC, Duncan BB, Schmidt MI. Prevalência de diabetes autorreferido no Brasil: resultados da Pesquisa Nacional de Saúde 2013. Epidemiol Serv Saude 2015; 24(2):305-314.

48. World Health Organization (WHO). Global health risks: mortality and burden of disease attributable to selected major risks. Geneva: WHO; 2009.

49. Rutstein DD, Berenberg W, Chalmers TC, Child 3rd CG, Fishman AP, Perrin EB. Measuring the quality of medical care. a clinical method. New Engl J Med 1976; 294(11):582-588.

50. Mackenbach J, Bouvier-Colle M, Jougla E. "Avoidable" mortality and health services: a review of aggregate data studies. J Epidemiol Community Health 1990; 44:106-111

51. Sousa NFS, Lima MG, Cesar CLG, Barros MBA. Envelhecimento ativo: prevalência e diferenças de gênero e idade em estudo de base populacional. Cad Saude Publica 2018; 34(11):e00173317.

Artigo apresentado em 23/06/2020

Aprovado em 05/10/2020

Versão final apresentada em 07/10/2020

Editores-chefes: Romeu Gomes, Antônio Augusto Moura da Silva 